\title{
A polarization model for the German Vacuum Tower Telescope from in situ and laboratory measurements ${ }^{\star}$
}

\author{
C. Beck ${ }^{1}$, R. Schlichenmaier ${ }^{1}$, M. Collados ${ }^{2}$, L. Bellot Rubio ${ }^{1,3}$, and T. Kentischer ${ }^{1}$ \\ 1 Kiepenheuer-Institut für Sonnenphysik, Schöneckstr. 6, 79104 Freiburg, Germany \\ e-mail: [cbeck;schliche; lbellot; tk]@kis.uni-freiburg.de \\ 2 Instituto de Astrofísica de Canarias, via Láctea, La Laguna, Tenerife, Spain \\ e-mail: mcv@ll.iac.es \\ 3 Instituto de Astrofísica de Andalucía, Apdo. 3004, 18080 Granada, Spain \\ e-mail: 1bellot@iaa.es
}

Received 25 February 2005 / Accepted 21 July 2005

\section{ABSTRACT}

It is essential to properly calibrate the polarimetric properties of telescopes, if one wants to take advantage of the capabilities of high precision spectro-polarimeters. We have constructed a model for the German Vacuum Tower Telescope (VTT) that describes its time-dependent polarization properties. Since the coelostat of the telescope changes the polarization state of the light by introducing cross talk among different polarization states, such a model is necessary to correct the measurements, in order to retrieve the true polarization as emitted from the Sun. The telescope model is quantified by a time-dependent Mueller matrix that depends on the geometry of the light beam through the telescope, and on material properties: the refractive indices of the coelostat mirrors, and the birefringence of the entrance window to the vacuum tube. These material properties were determined experimentally in-situ by feeding the telescope with known states of polarization (including unpolarized light) and by measuring its response, and from measurements of an aluminum-coated sample in the laboratory. Accuracy can in our case be determined only for the combination of telescope and spectro-polarimeter used; for the instrument POLIS at the VTT, we estimate an accuracy of $\pm 4-5 \times 10^{-3}$ for the cross talk correction coefficients.

Key words. instrumentation: polarimeters - sun: magnetic fields

\section{Introduction}

The light of absorption and emission lines can be polarized through various atomic processes, e.g., the Zeeman and Hanle effect for magnetic fields. Continuum polarization can be introduced by scattering processes with a preferred illumination direction, or by synchrotron and cyclotron radiation. Thus, the information contained in the polarization state of light allows detailed insight into the physical processes in astrophysical objects, or into their geometry. To access this information, the polarization state has to be measured via remote sensing, i.e. by analyzing the electromagnetic waves which are emitted by the sources.

Polarimeters have been standard instruments at solar telescopes since Hale's discovery that sunspots are manifestations of strong magnetic fields. Due to the easy availability of high-quality polarization optics and of CCD detectors with high quantum-efficiency, polarimeters for the visible or nearinfrared spectrum are becoming more frequent in, e.g., stellar and galactical physics as well.

\footnotetext{
* Appendices are only available in electronic form at
} http://www.edpsciences.org
In this contribution we use the German Vacuum Tower Telescope on Tenerife (VTT), a solar telescope, as an example. The VTT is equipped with two spectro-polarimeters that measure the polarization properties at high spatial and spectral resolution: the Tenerife Infrared Polarimeter (TIP, Martínez Pillet et al. 1999), which can be used for almost any line in the near infrared; the Polarimetric Littrow spectrograph (POLIS), which simultaneously measures two neutral iron lines in the visible at $630.2 \mathrm{~nm}$, and the Ca II H line in the near-UV at $397 \mathrm{~nm}$ (Schmidt et al. 2001; Beck et al. 2005).

For high precision polarimetric measurements, it is essential to correct for the cross talk among different polarization states which is introduced by reflections on metallic surfaces in the telescope in front of the polarimeter. In this contribution, we describe and then verify an analytical model for the polarimetric properties of a single aluminum-coated mirror surface that uses the complex refractive index of the material as an input parameter. The approach presented can be adjusted to other telescopes for the description of their polarimetric properties; this may be useful if no in-situ measurements are possible, because the refractive index can be measured in an optical laboratory on a sample mirror. We applied the formalism to 
the optical train of the VTT to derive a time-dependent model of its polarization properties for infrared and visible wavelengths. Polarization models for other solar telescopes have been presented by Balasubramaniam et al. (1985), Capitani et al. (1989), Skumanich et al. (1997), and Bernasconi (1997). Horn et al. (1996) present a Mueller matrix of the VTT for some fixed times (without a model), but it includes both telescope and polarimeter, which makes comparison difficult.

The VTT uses a coelostat to feed the solar light into the telescope. While this guarantees a non-rotating image in the focal plane as the Sun changes its position on the sky, the beam geometry changes during the day and during the year. Hence, it is necessary to develop a model which allows prediction of the polarization properties for a specific configuration. This model is described in Sect. 2. As we will show, the two essential unknowns of this model are the complex refractive index of the two coelostat mirrors and the birefringence of the entrance window. In Sect. 3 we use polarizing sheets to determine the properties of the window. The refractive index is determined in Sect. 4 by two methods: direct measurements in the laboratory (cf. Sect. 4.1) and derivation from the continuum polarization induced by the telescope (Sect. 4.2). In Sect. 5 we present typical results for the Mueller matrix of the telescope, discuss the corresponding errors, and describe the polarimetric calibration procedure as it is applied to TIP and POLIS data.

\section{The telescope model}

A sketch of the light beam geometry is displayed in Fig. 1. The coelostat consists of two flat mirrors ( $\mathrm{C} 1$ and $\mathrm{C} 2$ ), which feed the beam into the telescope. The second mirror, $\mathrm{C} 2$, is mounted on a pillar. Its height depends on the Sun's declination and on the position of $\mathrm{C} 1$. $\mathrm{C} 1$ can be moved on a circle around $\mathrm{C} 2$ while its axis is parallel to the earth rotation axis, e.g., to avoid the shadow of $\mathrm{C} 2$ falling on $\mathrm{C} 1$. The displacement of $\mathrm{C} 1$ from the North-South direction is measured by the angle $\gamma$, which by convention is positive towards the east. $\mathrm{C} 1$ is rotated to follow the Sun's track on the sky; i.e., only the angle of incidence on $\mathrm{C} 1, i_{\mathrm{C} 1}$, is time-dependent for a given position of $\mathrm{C} 1$ and $\mathrm{C} 2$. The small changes in solar declination during one day produce negligible variations of the angle of incidence on $\mathrm{C} 2, i_{\mathrm{C} 2}$.

The imaging main mirror, M3, and a folding flat mirror, M4, are contained in a vacuum tube with an entrance and an exit window made of BK7 glass. Just below the exit window the Instrument Calibration Unit (ICU) is installed to calibrate the polarization properties of the remaining light path: the optics of the correlation tracker (CT) system ${ }^{1}$ and the instrument itself. The polarimetric calibration of this part of the beam and of the spectro-polarimeter by its response function is described by Beck et al. (2005) for POLIS, and by Schlichenmaier \& Collados (2002) and Collados (2003) for TIP.

In the following, the system of M3, M4, and the two windows will be referred to as "telescope proper", while the term "whole telescope" also includes the coelostat.

\footnotetext{
${ }^{1}$ Since the beginning of 2004, the CT has been replaced by the Kiepenheuer Adaptive Optics System (KAOS, von der Lühe et al. 2003).
}

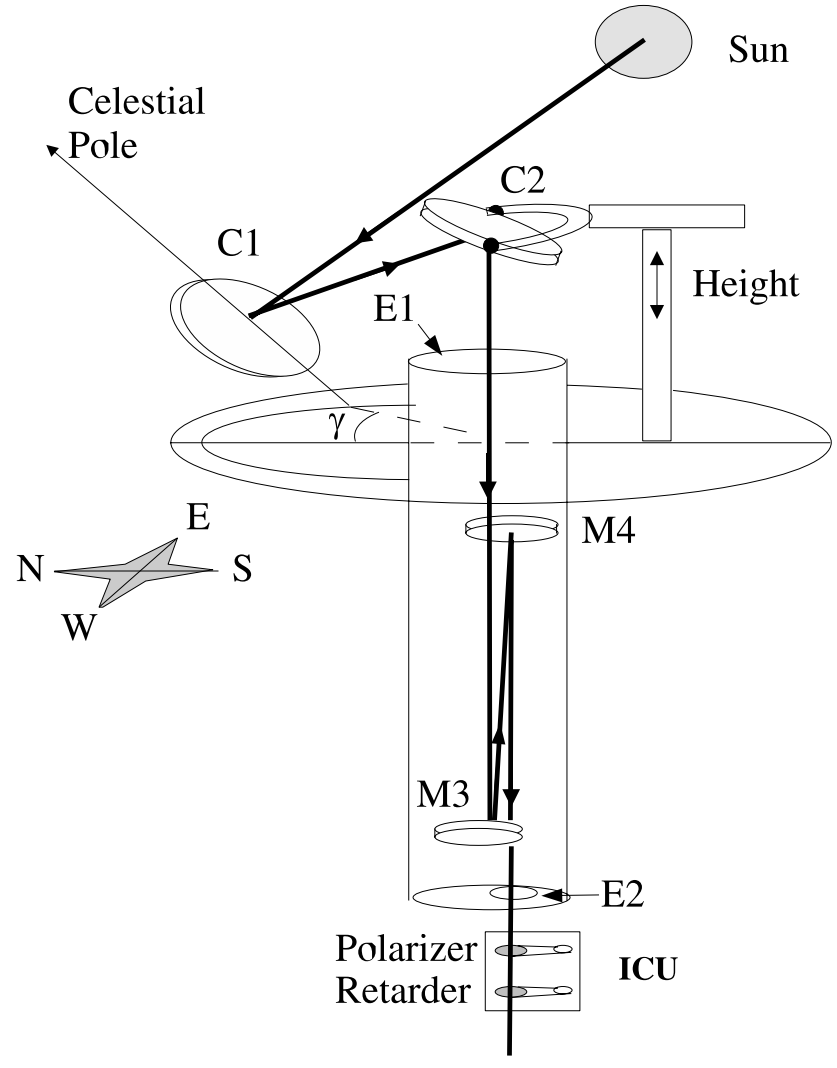

Fig. 1. Beam geometry of the VTT (sketch not to scale). The coelostat consists of two flat aluminum-coated mirrors ( $\mathrm{C} 1$ and $\mathrm{C} 2)$, which feed the light beam into the telescope. M3 is the imaging mirror $(f=46 \mathrm{~m})$. M4 folds the light down to the laboratories. E1 and E2 are the entrance and exit windows of the evacuated tube. The Instrument Calibration Unit (ICU) is located below the exit window.

\subsection{Mueller matrix of the whole telescope}

The polarization state of light is commonly described in the Stokes formalism (Shurcliff 1966; Collett 1992). The polarization state is expressed by the four Stokes parameters $I, Q, U$, and $V$, which are usually combined into the Stokes vector, $S$. $I$ measures intensity, $Q$ and $U$ measure the content of linear polarization at different angles, and $V$ the amount of circular polarization. The change of the Stokes vector due to an optical element corresponds to a transformation of the vector. It is described using a Mueller matrix, $\mathcal{M}$, by $\boldsymbol{S}_{\text {out }}=\mathcal{M} \cdot \boldsymbol{S}_{\text {in }}$. By convention the first index of an individual matrix entry, $\mathcal{M}(i, k)$ $(i, k=1 \ldots 4)$, denotes rows and the second columns.

For the telescope model, three effects have to be described quantitatively by Mueller matrices: reflection on a mirror, rotation of the reference frame for the linear polarizations, and transmission through a retarder.

The mirrors at the VTT have plain aluminum coatings with no additional protective layer. The coating device is set up for a coating thickness of $1 \mu \mathrm{m}$. For the description of the mirrors, we have adopted a simplified version of the equations for reflection on a metallic surface layer given by Skumanich et al. (1997). We assume (and justify a posteriori) that our aluminum coatings are sufficiently thick for the material properties of the mirror substrate below the coating to have no effect on the 
reflection. The same applies to the existence of a thin aluminum oxide layer on top of it which forms after the coating process. Three parameters are then needed to calculate the Mueller matrix of a mirror: the angle of incidence, $i_{\mathrm{M}}$, and two material properties, the index of refraction, $n_{\lambda}$, and the index of extinction, $k_{\lambda}$. The modified equations are given in Appendix A.

The glass of the windows to the evacuated telescope tube is under tension and might change the polarization state of the beam. In Sect. 3 we argue that the windows can be described as an ideal retarder with an effective retardance, $\Delta$, and an orientation angle of the fast optical axis, $\alpha$, which shows a daily variation.

The Mueller matrix of the telescope, $\mathcal{T}$, results from subsequently multiplying individual Mueller matrices as the beam passes through the telescope:

$$
\begin{aligned}
\boldsymbol{S}_{\text {out }} & =\mathcal{T} \cdot \boldsymbol{S}_{\mathrm{in}} \\
& =\boldsymbol{W}_{\mathrm{E} 2} \cdot \mathcal{R}\left(\theta_{4}\right) \cdot \mathcal{M}_{\mathrm{M} 4} \cdot \mathcal{M}_{\mathrm{M} 3} \cdot \mathcal{W}_{\mathrm{E} 1} \cdot \mathcal{R}\left(\theta_{3}\right) \\
& \cdot \mathcal{M}_{\mathrm{C} 2} \cdot \mathcal{R}\left(\theta_{2}\right) \cdot \mathcal{M}_{\mathrm{C} 1} \cdot \mathcal{R}\left(\theta_{1}\right) \cdot \boldsymbol{S}_{\mathrm{in}} .
\end{aligned}
$$

$\mathcal{R}\left(\theta_{i}\right)$ denotes rotations of the reference frames between subsequent optical elements, where $\mathcal{R}\left(\theta_{1}\right)$ rotates the fixed reference system on the Sun onto the first mirror. We define the reference frame on the Sun by the great circle through Sun center and the celestial pole. Positive $Q$ is parallel to the tangent to the circle at Sun center.

The Mueller matrix of the telescope depends (a) on the geometry of the beam (the angles of incidence on the 4 mirrors, $i_{\mathrm{C} 1}(t), i_{\mathrm{C} 2}, i_{\mathrm{M} 3}$, and $i_{\mathrm{M} 4}$, the rotation angles, $\left.\theta_{1}(t), \theta_{2}, \theta_{3}, \theta_{4}\right)$ and (b) on material properties, i.e. the refraction indices, $n$, and the extinction coefficients, $k$, of the mirrors, and the effective phase shift, $\Delta$, and angle of the optical axis, $\alpha$, of the windows. While the geometry of the beam is deduced in the next subsection, the material properties are determined by measurements that are described in Sects. 3 and 4.

\subsection{Construction of the coelostat beam geometry}

The beam geometry of a coelostat is described and solved analytically by Capitani et al. (1989) (C89 hereafter). As they demonstrate, the light path is fixed, once the positions of the Sun and of the second mirror, C2, relative to the first mirror, $\mathrm{C} 1$, are given. The equations of $\mathrm{C} 89$ allow derivation of the angles of incidence on $\mathrm{C} 1$ and $\mathrm{C} 2, i_{\mathrm{C} 1}$ and $i_{\mathrm{C} 2}$, and the rotation angles $\theta_{1}$ and $\theta_{2}$. The derivation and adjustment to the geometry of the VTT is given in Appendix C.

The beam path in the telescope proper is fixed by design, and the necessary values are:

$$
\begin{aligned}
i_{\mathrm{M} 3} & =i_{\mathrm{M} 4}=0.85^{\circ} \\
\theta_{3} & =90^{\circ}-\gamma \\
\theta_{4} & =90^{\circ} .
\end{aligned}
$$

Now, all angles of incidence and the rotation angles needed to evaluate the Mueller matrix of the telescope are known; i.e. for a given date and time the beam geometry is given. We are left with determining the material properties that need to be plugged into the telescope model.

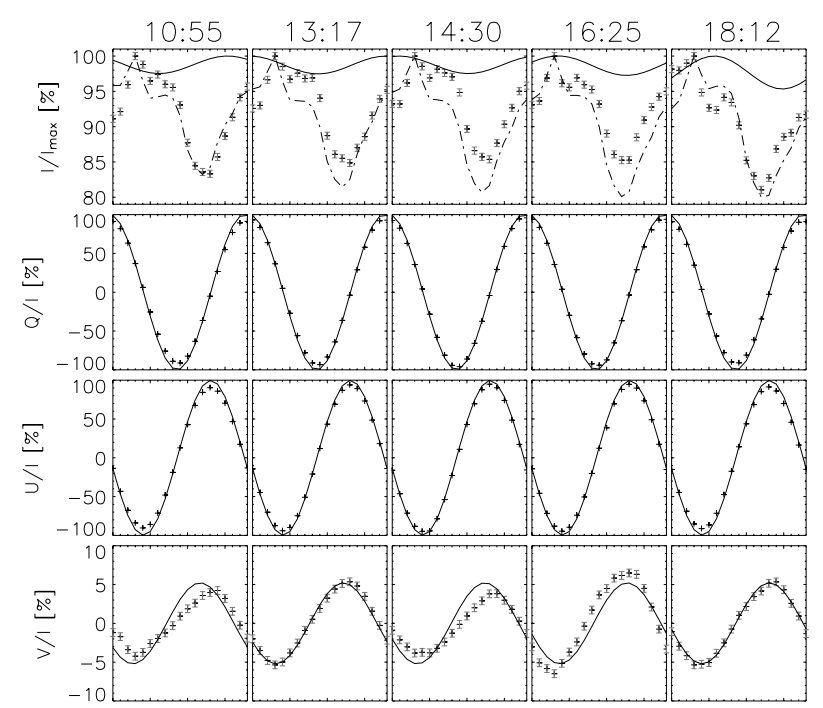

Fig. 2. Response of the telescope proper to linearly polarized light at $630 \mathrm{~nm}$, as the telescope calibration unit was rotated by $180^{\circ}$ in steps of $10^{\circ}$. From top to bottom the four Stokes parameters are shown for 5 different times during the day.

\section{Properties of the telescope proper}

In order to derive the response of the telescope proper, i.e., E1, M3, M4, and E2, two telescope calibration units (TCUs) were built for infrared and ultraviolet wavelengths. The TCUs consist of arrays of rectangular foils, which transmit linearly polarized light. The polarization axes of the individual foils were all aligned parallel to each other. To obtain a calibration data set, the TCU is located in front of the entrance window and is rotated by $180^{\circ}$ in steps of $10^{\circ}$, i.e. 19 measurements are made. The measurements are either performed with TIP or with POLIS.

Figure 2 displays five data sets that were recorded with POLIS on July 27, 2003, with $\mathrm{C} 1$ at $\gamma=0^{\circ}$, using the infrared TCU. From top to bottom the four Stokes parameters $I / I_{\max }, Q / I, U / I$, and $V / I$ are shown for 5 different times from the morning (left) to the evening (right). The crosses mark the 19 measurements obtained for each data set. As a comparison, the solid lines correspond to the results from the telescope model (see below). At zero degrees the light input consists of pure $Q$ signal, at $45^{\circ}$ of pure $-U$ and so on. The measured $Q$ and $U$ signals are in phase with the input signal. Two apparent features need to be discussed below: (a) The Stokes $I$ dataset exhibits an irregular pattern, and (b) the telescope proper produces up to $5 \%$ of Stokes $V$. The maximum $V$ signal is reached where $U$ is almost at the maximum, but its position is slightly changing during the day. In the measurements shown in Fig. 2 the polarization degree, $p=\sqrt{Q^{2}+U^{2}+V^{2}} / I$, is only about $95 \%$. As the depolarization effect of the entrance window should be negligible (cf. Skumanich et al. 1997), we believe this to be a property of the polarizer foils, which have been specified for infrared wavelengths but are used in the visible spectral range. As a check we derived the Mueller matrix of one of the polarizing foils by placing the foil between the Instrument Calibration Unit and the polarimeter itself. 
The matrix also yielded a transmission of some $5 \%$ of unpolarized light.

Modulation of $I$ : the irregular pattern of Stokes $I$ is due to the spider mounting of the mirror that feeds the guiding telescope of the VTT. Since the TCU is composed of individual rectangles, the transmitted light varies as these rectangles are rotated over the spider and sometimes are partially obscured by it. A crude modelling of this varying light level results in the dashdotted curve in the figure. It reproduces the measurement fairly well, but agreement is not good enough to use the modulation of $I$ to estimate the polarization introduced by the coelostat. Without the spider, one would be able to determine the amount of linearly polarized light produced by the coelostat from the modulation of $I$ as the polarizers are rotated on the entrance window. This expected modulation of $I$ is shown with the solid lines in the upper panel of Fig. 2. Note that the model modulation of $I$ shows a phase shift with time (as the coelostat angles change during the day) while the irregular (spider) pattern of $I$ does not change with time, reassuring that the irregular pattern is a geometric effect. For this reason, the variation of $I$ is not used to calibrate the coelostat; only the ratios $Q / I, U / I$, and $V / I$ are used.

Modelling the generation of $V$ : the prime candidates for introducing birefringence are the entrance and exit window, as the mirrors within the telescope are almost at normal incidence. The evacuated telescope exerts stress on the entrance and exit windows. For a rotationally symmetric stress distribution, Owner-Petersen (1991) showed that no net retardance exists and that the Mueller matrix has only diagonal elements. This remains valid if the temperature of the window is not constant, but shows a radial gradient, still rotationally symmetric. Nonetheless, our measurements reveal that some $5 \%$ of $V$ is produced by the telescope proper. This finding can be modelled as an ideal retarder with small retardance. In Appendix B we demonstrate that such a retardance could be due to a window which suffers from a uni-directional stress distribution. We point out that it might be possible that different effects are present which produce birefringence; e.g., both the entrance and the exit window may contribute, but it was shown by Stenflo (1991) that a combination of retarders act as an effective ideal retarder, as long as each of them is only weakly polarizing. Hence we model the telescope proper as an ideal retarder, $B(\Delta)$, with the Mueller matrix given by Eq. (B.4). The effective retardance $\Delta$ is derived from the maximum amplitude of $V$, when the TCU is rotated on the entrance window; and the angle of the optical axis, $\alpha$, is determined from the direction of the linear polarizers in the TCU at which maximum $V$ is achieved.

As the solid lines in Fig. 2 demonstrate, measurements with the TCU can be reproduced by the telescope model. The Mueller matrix of the telescope proper is calculated with Eq. (B.5) with an effective retardance of $\Delta=3^{\circ}$ at $630 \mathrm{~nm}$, and an angle for the fast optical axis that varies slightly from $\alpha=20^{\circ}$ at 8:00 UT to $\alpha=0^{\circ}$ at 20:00 UT. From similar measurements with TIP in the infrared, a retardance value of $1.8^{\circ}$ at $1.5 \mu \mathrm{m}$ was found which is in reasonable agreement with a $1 / \lambda$ dependence of the retardance value. The telescope proper thus can be reproduced by an ideal retarder with a small retardance. In order to assure this behaviour, the measurements and the corresponding calibration are repeated regularly.

\section{Properties of the coelostat}

As a next step we determined the refraction index, $n$, and the absorption coefficient, $k$, of the coelostat mirrors. Since both mirrors are re-coated at least once a year, it is assumed that their material properties are identical. In a first step, we examined a sample that was coated together with one of the two mirrors. We determined its response to polarized light in the laboratory (Sect. 4.1). In the next step we took advantage of the unpolarized continuum radiation from the Sun and measured the polarization that is induced by the coelostat for different beam geometries.

\subsection{Direct measurement of sample mirror}

The response of the sample mirror to unpolarized light and to all types of linearly polarized light (using combinations with one or two polarizers in front and behind the mirror) was measured for reflections under varying angles of incidence. This allowed us to determine all matrix entries that do not deal with circular polarization.

The measurements were performed for three different wavelengths: $518 \mathrm{~nm}, 630 \mathrm{~nm}$, and $777 \mathrm{~nm}$. The measurements are displayed in Fig. 3, together with the resulting leastsquares-fits for the refraction index, $n$, and the extinction coefficient, $k$. The errors due to inaccurate adjustments of the angle of incidence and the axes of the polarizers result in a fit uncertainty for $(n, k)$ of $\pm(0.1,1)$. Within the error bar our findings compare well with the values given by Lide (1993) for aluminum (cf. Table 1). Hence, we confirm that the coating behaves like aluminum, and we justify a-posteriori the assumptions made in Sect. 2.1. The aluminum coating is sufficiently thick for the mirror substrate to have no influence on the properties of the mirror, and we do not see effects due to a possible aluminum oxide layer on top of the coating.

\subsection{Telescope response to unpolarized light}

The angle of incidence of the light on the first coelostat mirror varies during the day. Hence, the amount of cross talk from intensity to polarization, $I \rightarrow Q U V$, also varies. Figure 4 shows the daily variation of continuum polarization measured on June 28,2003 . The data were taken at disk center.

In order to reproduce the daily variation, $(n, k)$ pairs can be fitted with the help of the telescope model. However, since the intensity transmission of the telescope is not measured, $n$ and $k$ cannot be fitted independently; the fit is only significant for one free parameter. The solid line in Fig. 4 uses $n=1.41$, while $k=7.6$ was determined from a least-squares fit to the data. The error bars are given by the estimated error of the polarimeter response function of $1.4 \times 10^{-3}$ (Beck et al. 2005). 

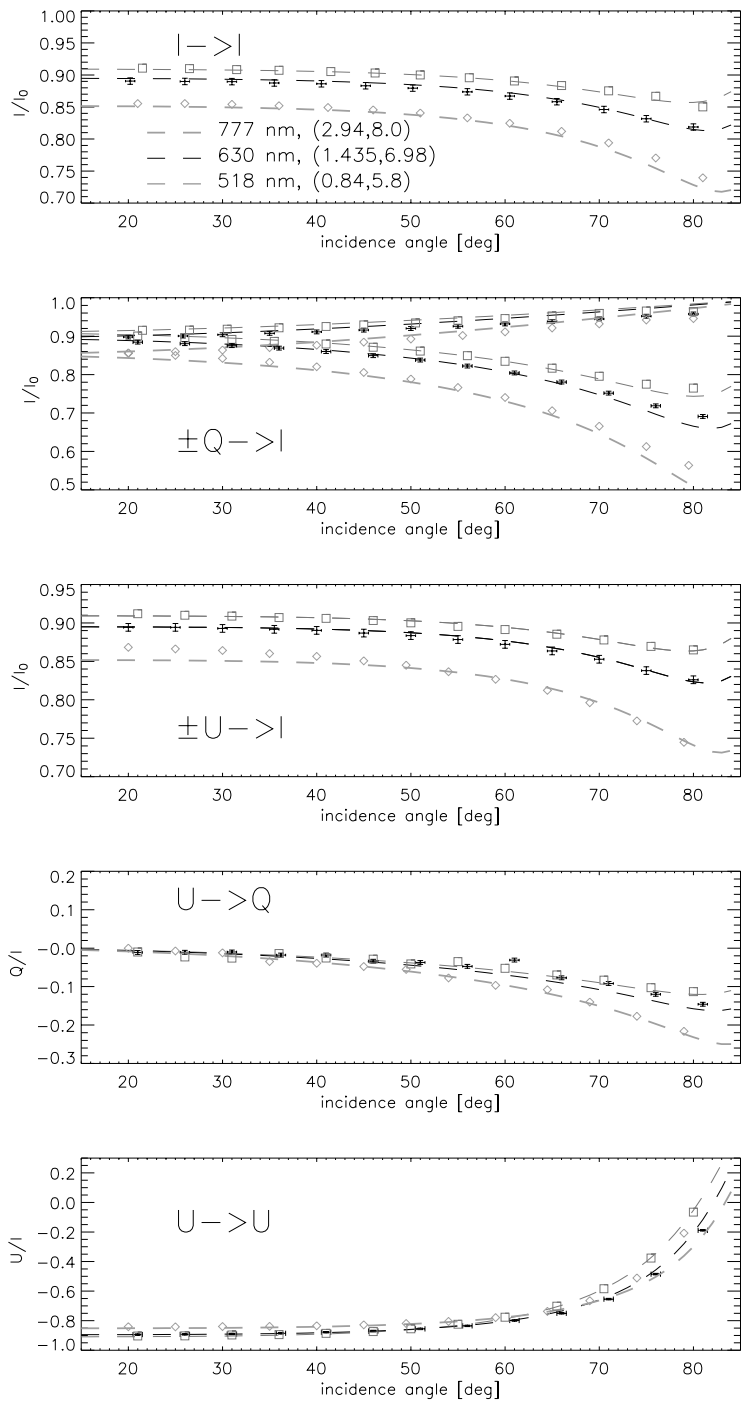

Fig. 3. For three wavelengths $(518 / 630 / 777 \mathrm{~nm})$, the Mueller matrix entries $\mathcal{M}(1,1), \mathcal{M}(1,2), \mathcal{M}(1,3), \mathcal{M}(2,3)$, and $\mathcal{M}(3,3)$ (top to bottom) of a mirror sample were measured as function of the angle of incidence, $i$. The measurements could be reproduced using the refractive indices given in the uppermost panel.

A second example is displayed in Fig. 5, where a "normal" solar observation was used, a repeated scan of a sunspot which covered about $1 \mathrm{~h}$. Note that the $(n, k)$ values found from Fig. 4 were used without further adjustment. On this time scale the variation of the continuum polarization can already be determined. Thus, it is possible to derive the properties of the coelostat mirrors from the continuum polarization even without taking special data for this purpose.

We believe that the measurements presented in this Sect. (1) prove the validity of the telescope model, including the description of the mirrors by the Eqs. of Appendix A, and (2) confirm the material properties that we assume for the coelostat mirrors, close to literature values for bulk aluminum. An additional control of the correct geometry of the telescope model is given in Appendix D using the well known image rotation of any coelostat for $\gamma \neq 0^{\circ}$.
Table 1. The complex refraction indices, $(n, k)$, for the bulk aluminum of a mirror sample were measured at three wavelengths. The best-fit values found from Fig. 3 (1 st row) agree with the corresponding values given by Lide (1993) (2nd row).

\begin{tabular}{lccc}
\hline \hline & $518 \mathrm{~nm}$ & $630 \mathrm{~nm}$ & $777 \mathrm{~nm}$ \\
\hline$(n, k)_{\text {meas }}$ & $(0.84,5.80)$ & $(1.44,6.98)$ & $(2.94,8.0)$ \\
$(n, k)_{\text {lit }}$ & $(0.83,6.30)$ & $(1.36,7.58)$ & $(2.63,8.59)$ \\
\hline
\end{tabular}
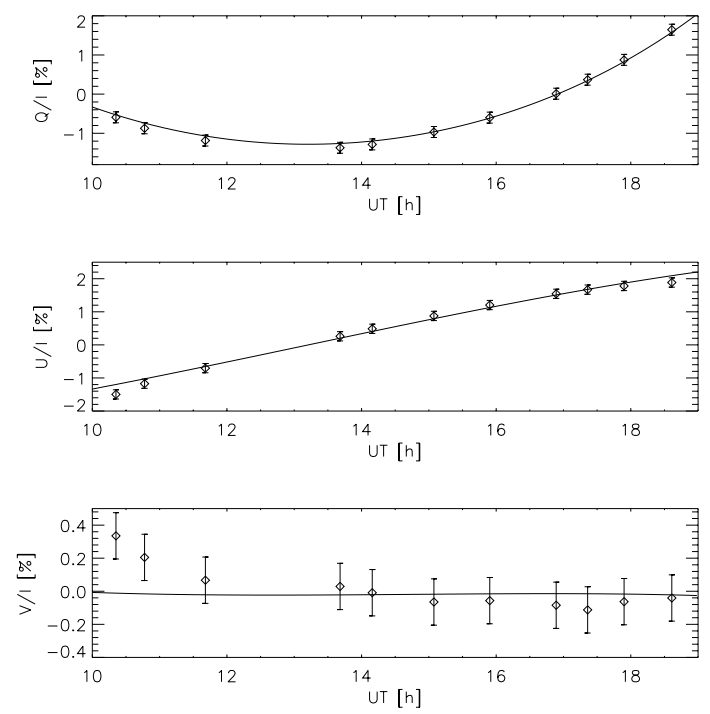

Fig. 4. The daily variation of the continuum polarization at $630 \mathrm{~nm}$ induced by the telescope on July 28, 2003 with $\mathrm{C} 1$ at $\gamma=0^{\circ}$. The solid curve corresponds to the prediction of the telescope model with $n=1.41$ and $k=7.6$.
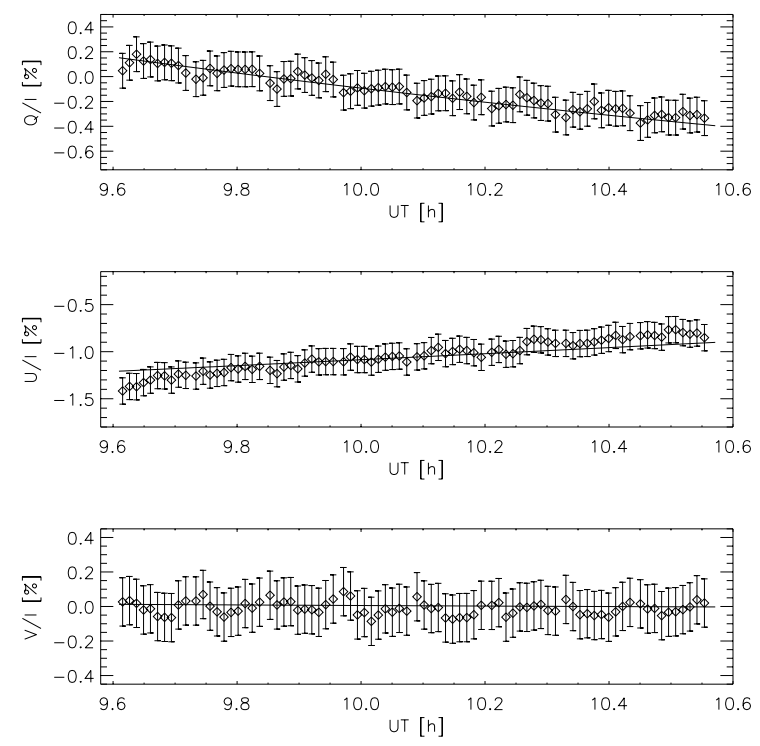

Fig. 5. Same as Fig. 4 for an observation of a sunspot on August 9, 2003, from UT 9:30 to $10: 30$.

\section{Telescope matrix}

Having determined the effective Mueller matrix of the telescope proper, the refraction and extinction coefficients of the coelostat mirrors, we are able to evaluate the telescope matrix 
for specific configurations, i.e. for a specified day and time and for a specified position of $\mathrm{C} 1$. As an example, the telescope matrix for March 21 at 10:00 UT $\left(\delta_{\odot} \sim 0^{\circ}, \gamma=50^{\circ}\right)$ at $630 \mathrm{~nm}$ is

$\mathcal{T}_{630 \mathrm{~nm}}=\left(\begin{array}{rrrr}1 & 0.0232 & -0.0053 & -0.0007 \\ 0.0238 & 0.9653 & -0.2591 & -0.0311 \\ 0.0009 & 0.2605 & 0.9495 & 0.1731 \\ -0.0001 & -0.0154 & -0.1752 & 0.9841\end{array}\right)$,

while in the infrared it reads

$\mathcal{T}_{1.5 \mu \mathrm{m}}=\left(\begin{array}{rrrr}1 & 0.0055 & -0.0013 & -0.0001 \\ 0.0057 & 0.9650 & -0.2622 & -0.0012 \\ 0.0001 & 0.2617 & 0.9631 & 0.0629 \\ -0.0001 & -0.0153 & -0.0610 & 0.9980\end{array}\right)$.

The matrix has been normalized with the intensity transmission, $\mathcal{T}(1,1)$, which was $68 \%$ at $630 \mathrm{~nm}$ and $78 \%$ in the infrared. For the visible spectral range, the values $(n, k)=(1.41$, 7.6) were used. For the infrared $(n, k)=(1.466,16.118)$ were interpolated from the tabulated numbers given by Lide (1993). The matrix for the infrared was calculated without the retardance of the entrance window (cf. Sect. 5.2). In the infrared, the telescope matrix shows generally lower cross talk and a higher total transmission. The matrix entries may exceed $20 \%$ in $V \rightarrow U$ earlier in the day (cf. Appendix E).

\subsection{Error estimate for the telescope model}

It is difficult to separate the error introduced by the telescope model from that due to the polarimeter response function. Errors of the telescope model lead mainly to a change in the shape of the curve of the daily or hourly variation (cf. Figs. 4 and 5). Errors in the response function can change the polarization level by a constant offset, but can also change the shape through the cross talk $Q U V \leftrightarrow Q U V$. We thus feel compelled to give an error estimate for the complete optical train, the combination of polarimeter and telescope, rather than separate numbers.

The deviation between measurements and predictions of the telescope model is on the order of $3 \times 10^{-3}$, as can be seen most clearly in the lowermost panel of Fig. 4, Stokes $V$, in the early morning. The measured Stokes $U$ shows deviations in the shape of the curve early in the morning and late in the evening. The combined error of the cross talk corrections for telescope and polarimeter is thus about $4-5 \times 10^{-3}$, and seems to depend on the light level in the telescope. It is assumed that all other matrix entries show deviations similar to those of the first column.

Note that the value given is the error in the determination of the cross talk coefficients among the different polarization states. The telescope polarization is a second order effect: the matrix entries are multiplied with the incoming Stokes vector, $\boldsymbol{S}_{\text {in }}$. For example, taking only the dominant $U \rightarrow V$ cross talk from the matrix in Eq. (5) one has

$V_{\text {out }}=(0.984 \pm 0.005) \cdot V_{\text {in }}+(-0.175 \pm 0.005) \cdot U_{\text {in }}$,

where usually $U_{\text {in }}$ or $V_{\text {in }}$ do not exceed $0.3 \cdot I_{\text {in }}$.

\subsection{Telescope calibration for TIP and POLIS}

Data sets that are acquired with the polarimeters TIP and POLIS are flatfielded and calibrated with the polarimeter response function. After that they are corrected for cross talk introduced by the telescope. This is done slightly different; for POLIS the telescope model includes the retardance of the telescope proper and models the daily variation of the orientation of the fast axis. For TIP the effect of the retardance is smaller, due to the larger wavelength; the telescope proper is modeled without a retardance. In this case the remaining inaccuracy is corrected by applying indirect calibration techniques, developed by Kuhn et al. (1994) (see also, Schlichenmaier \& Collados 2002; Collados 2003). The Zeeman components of magnetic sensitive lines in the infrared are fully separated, at least in sunspots. In the transverse Zeeman effect where the amplitudes of $Q$ and $U$ are much larger than the $V$ amplitude, cross talk from $Q$ and $U$ to $V$ is easily detected. The same is true for cross talk from $V$ to $Q$ and $U$ for the longitudinal Zeeman effect. Since both configurations are present in sunspots, the method is ideally suited to determining residual cross talk. Since several, i.e. all suited, profiles are used by the method, it is referred to as the statistical cross talk correction. Although the performance of the method depends somewhat on the signal-to-noise ratio, it is very reliable in the infrared where the Zeeman splitting is sufficiently large.

\section{Summary}

New generation spectro-polarimeters like the Tenerife Infrared Polarimeter (TIP), the POlarimetric Littrow Spectrograph (POLIS), and in the future also the Triple Etalon SOlar Spectrometer (TESOS, Kentischer et al. 1998; Tritschler et al. 2002) are capable of performing high precision polarimeteric measurements. To make full use of their capabilities it became essential to model the polarization properties of the German Vaccuum Tower Telescope on Tenerife, such that the solar Stokes parameters can be retrieved properly from the measured ones. In Sect. 2 we develop an analytical model for the polarization properties of the telescope, which represents a modification of the model from the Arcetri observatory by Capitani et al. (1989). The analytical model depends on the Mueller matrices of the individual optical components.

The telescope proper, consisting of the main mirror, a flat mirror, and an entrance and exit window to the vacuum, is described as an effective Mueller matrix. In Sect. 3 the polarizing properties of the telescope proper are determined by feeding the telescope with linearly polarized light of varying directions, and by measuring the response with TIP and POLIS. We find that the telescope proper exhibits a retardance of some $3^{\circ}$ at $630 \mathrm{~nm}$, with a slightly changing orientation of the respective optical axis. We believe that this change of orientation reflects the non-uniform radiation from the Sun on the upper end of the telescope tube, which will pass from east to west during the day, leading to a uni-directional stress in the entrance window through thermal expansion of its mounting.

The other free model parameters, namely, the refraction and extinction coefficients, $(n, k)$, of the coelostat mirrors are 
determined in Sect. 4. The aluminum coating is best described with a one-layer reflection that has the indices $(n, k)=$ $(1.4-1.45,7-7.6)$ at $630 \mathrm{~nm}$. The values are in agreement with literature values for bulk aluminum, and were derived consistently by two independent methods: measurements of a sample mirror in the laboratory and in-situ measurements at the telescope site using POLIS. Confidence in the validity of the telescope model and the model parameters used is given by the demonstration that the daily variation of the continuum polarization, only due to the telescope, is reproduced to within $0.3 \%$. Two examples of the telescope Mueller matrix are presented in Sect. 5 demonstrating that the application of the telescope model to polarimetric data is essential to retrieve reliable measurements.

It is possible to derive the refractive indices of sample mirrors with a simple laboratory measurement setup. With the values of $n$ and $k$, the polarimetric properties of the mirror can be calculated for any beam geometry with an accuracy that is sufficient for a polarimetric calibration of observation data, even if no in-situ measurements are possible at the telescope site.

\footnotetext{
Acknowledgements. We thank W. Schmidt and A. Bitzer for advice and support. We are grateful to D. Soltau for making us aware of the spider shadow. This work was supported by the Deutsche Forschungsgemeinschaft under grant Schl 514/2-1. This research was partly funded by the Spanish Ministerio de Educación y Ciencia through projects AYA2004-05792, ESP2003-07735-C04-03, and Programa Ramón y Cajal.
}

\section{References}

Balasubramaniam, K. S., Venkatakrishnan, P., \& Bhattacharyya, J. C. 1985, Sol. Phys., 99, 333
Beck, C., Schmidt, W., Kentischer, T., \& Elmore, D. 2005, A\&A, 437, 1159

Bernasconi, P. N. 1997, Ph.D. Thesis

Capitani, C., Cavallini, F., Ceppatelli, G., et al. 1989, Sol. Phys., 120, 173

Collados, M. 2003, in Polarimetry in Astronomy, ed. S. Fineschi, Proc. SPIE, 4843, 55

Collett, E. 1992, Polarized light. Fundamentals and applications, Optical Engineering (New York: Dekker)

Horn, T., Hofmann, A., \& Balthasar, H. 1996, Sol. Phys., 164, 321

Kentischer, T. J., Schmidt, W., Sigwarth, M., \& Uexkuell, M. V. 1998, A\&A, 340, 569

Kuhn, J. R., Balasubramaniam, K. S., Kopp, G., et al. 1994, Sol. Phys., 153,143

Lide, D. 1993, CRC Handbook of chemistry and physics (74th edition) Martínez Pillet, V., Collados, M., Sánchez Almeida, J., et al. 1999, in High Resolution Solar Physics: Theory, Observations, and Techniques, ASP Conf. Ser., 183, 264

Owner-Petersen, M. 1991, in LEST Technical Rep. No. 49, ed. O. Engvold, \& O. Hauge, Institute of Theoretical Astrophysics, University of Oslo

Schlichenmaier, R., \& Collados, M. 2002, A\&A, 381, 668

Schmidt, W., Kentischer, T. J., Bruls, J., \& Lites, B. W. 2001, in Advanced Solar Polarimetry - Theory, Observation, and Instrumentation, ASP Conf. Ser., 236, 49

Shurcliff, W. A. 1966, Polarized light. Production and use (Cambridge, Mass.: Harvard University Press)

Skumanich, A., Lites, B. W., Martínez Pillet, V., \& Seagraves, P. 1997, ApJS, 110, 357

Stenflo, J. 1991, in LEST Technical reports No. 45, ed. O. Engvold, \& O. Hauge, Institute of Theoretical Astrophysics, University of Oslo

Tritschler, A., Schmidt, W., Langhans, K., \& Kentischer, T. 2002, Sol. Phys., 211, 17

von der Lühe, O., Soltau, D., Berkefeld, T., \& Schelenz, T. 2003, in Innovative Telescopes and Instrumentation for Solar Astrophysics, ed. L. StephenKeil, \& V. Sergey Avakyan, Proc. SPIE, 4853, 187 


\section{Online Material}




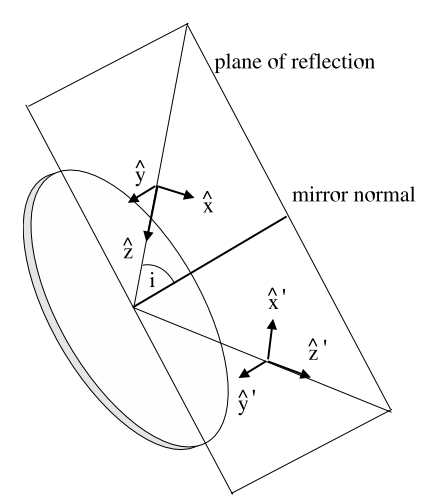

Fig. A.1. Sketch of reflection on a flat mirror. The incoming ray is described in the right-handed orthogonal coordinate system given by $\{\hat{x}, \hat{y}, \hat{z}\}$, the reflected beam in the primed one. $\hat{x}$ and $\hat{x}^{\prime}$ lie in the incidence-reflection plane, while $\hat{y}$ and $\hat{y}^{\prime}$ are parallel to the mirror surface. $i$ denotes the angle of incidence.

\section{Appendix A: Mueller matrix of mirrors}

Figure A.1 sketches the reflection on a mirror. The Stokes vector of the incoming beam defined in the reference frame $\{\hat{x}, \hat{y}, \hat{z}\}$ is transformed into the Stokes vector of the outgoing beam in the reference system $\left\{\hat{x}^{\prime}, \hat{y}^{\prime}, \hat{z}^{\prime}\right\}$. The two reference systems are defined such that $\hat{z}$ is pointing in the direction of propagation, $\hat{x}$ is in the incidence plane and is directed towards the mirror normal, and $\hat{y}$ is parallel to the mirror plane. The corresponding Mueller matrix which transforms the Stokes vector from basis $\{\hat{x}, \hat{y}, \hat{z}\}$ to $\left\{\hat{x}^{\prime}, \hat{y}^{\prime}, \hat{z}^{\prime}\right\}$ is given by (cf. Skumanich et al. 1997):

$$
\begin{aligned}
& \mathcal{M}\left(i_{\mathrm{M}}, n, k\right)= \\
& \left(\begin{array}{cccc}
\frac{\left(r_{x}+r_{y}\right)}{r^{2}} & \frac{\left(r_{x}-r_{y}\right)}{2} & 0 & 0 \\
\frac{\left(r_{x}-r_{y}\right)}{2} & \frac{\left(r_{x}+r_{y}\right)}{2} & 0 & 0 \\
0 & 0 & \sqrt{r_{x} r_{y}} \cos \delta & \sqrt{r_{x} r_{y}} \sin \delta \\
0 & 0 & -\sqrt{r_{x} r_{y}} \sin \delta & \sqrt{r_{x} r_{y}} \cos \delta
\end{array}\right),
\end{aligned}
$$

where $r_{x}$ and $r_{y}$ are the intensity reflection coefficients, and $\delta$ is the phase change, induced by the reflection, between the electric field components in the $\hat{x}^{\prime}$ and $\hat{y}^{\prime}$ direction. Under the assumption that the aluminum coatings are sufficiently thick such that the reflection is described as a one-layer reflection ${ }^{2}$, the three parameters $-r_{x}, r_{y}$, and $\delta-$ are related to the angle of incidence, $i_{\mathrm{M}}$, and two material properties: the index of refraction, $n_{\lambda}$, and the index of extinction, $k_{\lambda}$. Then, supressing the wavelength subscript for $n_{\lambda}$ and $k_{\lambda}$ for simplicity, the relations are given by

$r_{\|}=\frac{(n+\mathrm{i} k)^{2} \cos i_{\mathrm{M}}-u-\mathrm{i} v}{(n+\mathrm{i} k)^{2} \cos i_{\mathrm{M}}+u+\mathrm{i} v}$,

$r_{\perp}=\frac{\cos i_{\mathrm{M}}-u-\mathrm{i} v}{\cos i_{\mathrm{M}}+u+\mathrm{i} v}$

where $u$ and $v$ are calculated by

$$
u=\sqrt{\frac{1}{2}\left[c+\sqrt{c^{2}+4 n^{2} k^{2}}\right]}
$$

\footnotetext{
2 This corresponds to a value of $t=0$ in Eq. (A1) in Skumanich et al. (1997).
}

$v=\sqrt{\frac{1}{2}\left[-c+\sqrt{c^{2}+4 n^{2} k^{2}}\right]}$

with $c=n^{2}-k^{2}-\sin ^{2} i_{\mathrm{M}}$. With $r_{\|}=\sqrt{r_{x}} \mathrm{e}^{\mathrm{i} \epsilon_{x}}, r_{\perp}=\sqrt{r_{y}} \mathrm{e}^{\mathrm{i} \epsilon_{y}}$, $\sqrt{r_{x} r_{y}} \cos \delta=\operatorname{Re}\left(r_{\|} r_{\perp}^{*}\right)$, and $\sqrt{r_{x} r_{y}} \sin \delta=-\operatorname{Im}\left(r_{\|} r_{\perp}^{*}\right)$ the Mueller matrix parameters are defined.

\section{Appendix B: Mueller matrix of a window with a unidirectional stress distribution}

Owner-Petersen (1991) has derived the Mueller matrix of an entrance window which suffers from a uni-directional stress distribution. It is calculated from the addition of the electric field amplitudes of the light rays passing through different areas in the pupil plane. The final Stokes vector after addition is compared to the incident unpolarized light to derive the Mueller matrix. We caution the reader that their result refers to the central point of the spatial point spread function for an axial image point for a telescope at the diffraction limit. However, the final matrix is in close agreement with our observational results. According to Owner-Petersen (1991), the Mueller matrix for uni-directional stress is given by:

$\mathcal{W}=\left(\begin{array}{cccc}1-\frac{1}{2} \kappa_{2} & -\frac{1}{2} \kappa_{2} c_{2 \phi_{0}} & -\frac{1}{2} \kappa_{2} s_{2 \phi_{0}} & 0 \\ -\frac{1}{2} \kappa_{2} c_{2 \phi_{0}} & 1-\frac{1}{2} \kappa_{2} & 0 & -\kappa_{1} s_{2 \phi_{0}} \\ -\frac{1}{2} \kappa_{2} s_{2 \phi_{0}} & 0 & 1-\frac{1}{2} \kappa_{2} & \kappa_{1} c_{2 \phi_{0}} \\ 0 & \kappa_{1} s_{2 \phi_{0}} & -\kappa_{1} c_{2 \phi_{0}} & 1-\frac{1}{2} \kappa_{2}\end{array}\right)$,

using

$\kappa_{1}=\frac{1}{\pi} \int_{0}^{2 \pi} \int_{0}^{1} \delta(r, \phi) r \mathrm{~d} r \mathrm{~d} \phi$

$\kappa_{2}=\frac{1}{\pi} \int_{0}^{2 \pi} \int_{0}^{1} \delta^{2}(r, \phi) r \mathrm{~d} r \mathrm{~d} \phi$,

with the local retardance, $\delta(r, \phi)$, and the polar coordinates of the window, $0<r<1$ and $0<\phi<2 \pi . \kappa_{1}$ gives the averaged retardance of the window. The orientation of the optical axis enters via $c_{2 \phi_{0}}=\cos \left(2 \phi_{0}\right)$ and $s_{2 \phi_{0}}=\sin \left(2 \phi_{0}\right)$, with $\phi_{0}$ being the angle between the $x$-axis and the optical axis. Choosing $\phi_{0}$ to be zero, $\mathcal{W}_{31}, \mathcal{W}_{13}, \mathcal{W}_{42}, \mathcal{W}_{24}$ vanish. We note that a window described by Eq. (B.1) does not depolarize the beam, since all diagonal elements are equal and $\mathcal{W}_{12}=\mathcal{W}_{21}, \mathcal{W}_{13}=\mathcal{W}_{31}$, $W_{14}=W_{41}$.

For small retardances, first order terms in $\delta$, i.e. in $\kappa_{1}$, dominate the second order terms in $\delta^{2}$, i.e. in $\kappa_{2}$. From the observations, no indications of linear polarizing properties are visible. Hence, all off-diagonal matrix elements proportional to $\kappa_{2}$ are neglected in the upper left half of the matrix containing the cross talk $I \leftrightarrow Q$. In order to satisfy the properties of a physical Mueller matrix, i.e. $I^{2} \geq Q^{2}+U^{2}+V^{2}, \kappa_{2}$ has to be kept in the lower right part of the matrix. Expressing $\kappa_{1}$ by $\sin \triangle, W_{33}$ and $\mathcal{W}_{44}$ have to be approximated by $\cos \triangle$, leading to:

$\mathcal{B}(\Delta)=\left(\begin{array}{cccc}1 & 0 & 0 & 0 \\ 0 & 1 & 0 & 0 \\ 0 & 0 & \cos \Delta & \sin \Delta \\ 0 & 0 & -\sin \Delta & \cos \Delta\end{array}\right)$,

with a retardance $\Delta$. Therefore in the limit of small retardance, a window with a uni-directional stress distribution can be described to first order as an ideal retarder. From our calibration 


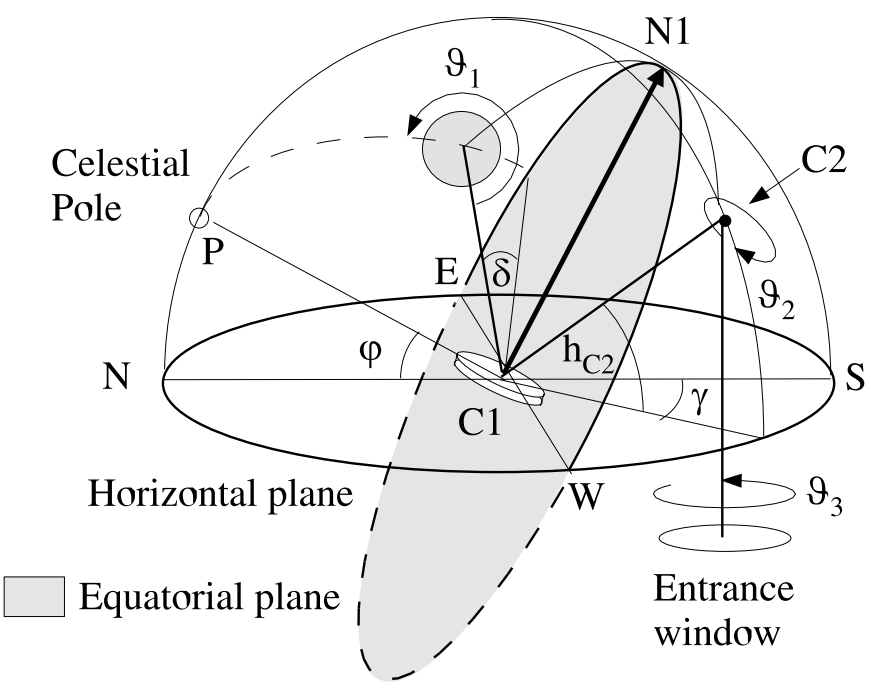

Fig. C.1. The coelostat in the equatorial and horizontal coordinate system. The origin is placed in the center of $\mathrm{C} 1$.

measurements, we find $\Delta=3^{\circ}=0.0523 \mathrm{rad}$, corresponding to $\sin \Delta=0.0524$ and $\cos \Delta \approx 1-1 / 2 \Delta^{2}=0.999$ which implies $1 / 2 \kappa_{2}=0.001$. These numbers both justify the assumption of a small retardance for the derivation of the Mueller matrix for the telescope proper, the neglect of the off-diagonal terms containing $\kappa_{2}$, and demonstrate that the window can be modelled as an ideal retarder to describe its polarimetric properties. The changing orientation of the optical axis is modelled by applying a rotation with an angle $\alpha$ :

$\mathcal{W}=\mathcal{R}(-\alpha) \mathcal{B}(\triangle) \mathcal{R}(\alpha)$

\section{Appendix C: Beam geometry of the coelostat}

The beam geometry of a coelostat is determined once the positions of the Sun on the sky and of the second mirror $(\mathrm{C} 2)$ relative to the first mirror $(\mathrm{C} 1)$ are given. The origin is defined by the center of $\mathrm{C} 1$. During the calculation, equatorial coordinates (declination, $\delta$, and hour angle, $H$ ) have to be transformed into the horizontal coordinate system (angular elevation above the horizon, $h$, and azimuth, $A$ ). The transformation is given by:

$$
\begin{aligned}
\cos h \cos A & =\cos \delta \cos H \sin \phi-\sin \delta \cos \phi, \\
\sin h & =\cos \delta \cos H \cos \phi+\sin \delta \sin \phi,
\end{aligned}
$$

where $\phi$ denotes the geographical latitude $\left(=28.30^{\circ}\right.$ at the German VTT in Tenerife).

The solar position in equatorial coordinates, $\left(\delta_{\odot}, H_{\odot}\right)$, is taken from an ephemerids table. The location of $\mathrm{C} 2$ relative to $\mathrm{C} 1$ is given by the angle $\gamma$ (cf. Fig. $1, \gamma>0$ if $\mathrm{C} 1$ is displaced to the east) and the angular height of $\mathrm{C} 2$ as seen from $\mathrm{C} 1, h_{\mathrm{C} 2}$.

For given values of $\gamma$ and $\delta_{\odot}$ the second mirror can only have one position to deflect the light into the telescope proper. Thus, $h_{\mathrm{C} 2}$ can be determined as follows: as sketched in Fig. C. 1 , the declination of $\mathrm{C} 2$ as seen from $\mathrm{C} 1$ in equatorial coordinates is $\delta_{\mathrm{C} 2}=-\delta_{\odot}$, and the azimuth of $\mathrm{C} 2$ in the horizontal system is $A_{\mathrm{C} 2}=\gamma$. The angular height of $\mathrm{C} 2, h_{\mathrm{C} 2}$, is determined by rewriting Eqs. (C.1) and (C.2) into:

$\sin \phi \sin h_{\mathrm{C} 2}-\cos \gamma \cos \phi \cos h_{\mathrm{C} 2}=\sin \delta_{\mathrm{C} 2}=-\sin \delta_{\odot}$.

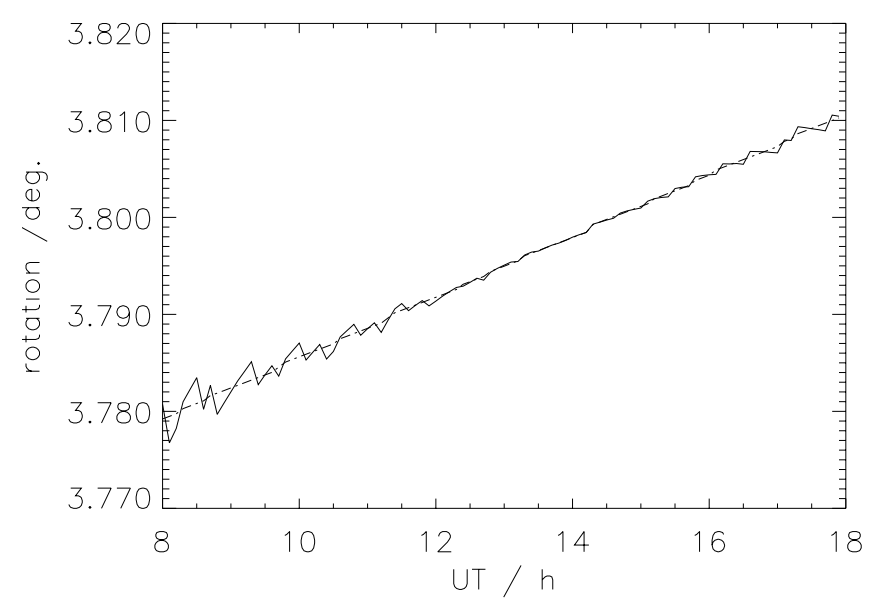

Fig. D.1. The change of the orientation angle of the linear polarization, $\beta_{\text {in }}-\beta_{\text {out }}$, during the day (solid line). The dash-dotted line is the prediction of Eq. (D.1) for the rotation angle. The increase in the rotation angle is caused by the changing declination of the Sun, while the differences are the result of numerical inaccuracies.

With a numerical solution for $h_{C 2}$ in Eq. (C.3), the hour angle of $\mathrm{C} 2, H_{\mathrm{C} 2}$, is given by:

$\sin H_{\mathrm{C} 2}=\cos h_{\mathrm{C} 2} \sin \gamma / \cos \delta_{\odot}$.

Given the configuration of the coelostat, the incidence and rotation angles can be evaluated using the sketch of Fig. C.1 and some trigonometry. Following C89, the angle of incidence of the beam on the first mirror is determined by

$\cos i_{\mathrm{C} 1}=\cos \delta_{\odot} \cos H$, with $H \equiv H_{\mathrm{N} 1}-H_{\odot}=\left(H_{\mathrm{C} 2}-H_{\odot}\right) / 2$, and hence $H_{\mathrm{N} 1} \equiv\left(H_{\mathrm{C} 2}+\right.$ $\left.H_{\odot}\right) / 2$. Note that only $H_{\odot}$ changes with time and that $H_{\mathrm{C} 2}$ is given by Eq. (C.4).

The rotation angle between the reference frames of the Sun to the first mirror is:

$\sin \theta_{1}=-\sin H / \sin i_{\mathrm{C} 1}$.

The angle of incidence on the second mirror is constant for a specified configuration of the coelostat, and is evaluated using

$i_{\mathrm{C} 2}=\left(90^{\circ}-h_{\mathrm{C} 2}\right) / 2$.

For the second rotation angle, one obtains

$\sin \theta_{2}=-\cos h_{\odot} \sin A / \sin 2 i_{\mathrm{C} 1}$,

with $A \equiv A_{\mathrm{C} 2}-A_{\odot}=\gamma-A_{\odot}$. The horizontal coordinates $h_{\odot}$ and $A_{\odot}$ are derived from the equatorial coordinates using Eqs. (C.1) and (C.2).

\section{Appendix D: Test of the telescope model: image rotation}

An additional option to test the correct geometry of the telescope model is the comparison of the physical image rotation, 
$p$, due to the coelostat at $\gamma \neq 0^{\circ}$ with the rotation of linear polarization in the model. The analytical calculation for the image rotation yields an angle

$p=-\operatorname{asin}\left(\frac{\cos \phi \sin \gamma}{\cos \delta_{\odot}}\right)+\gamma$

for the physical rotation.

For the linear polarization, the angle $\beta=1 / 2$ atan $(Q / U)$ can be calculated for the initial, $S_{\text {in }}$, and final Stokes vector, $\boldsymbol{S}_{\text {out }}=\mathcal{T} \cdot \boldsymbol{S}_{\text {in }}$. The difference $\beta_{\text {in }}-\beta_{\text {out }}$ must be identical to $p$ : $\beta_{\text {in }}$ is measured with respect to celestial N-S (first reference frame (RF), fixed on the Sun), but $\beta_{\text {out }}$ is measured with respect to terrestrial N-S (last RF, fixed in space). The physical image rotation rotates the celestial $\mathrm{N}$-S-direction in the focal plane by $p$ away from terrestrial N-S, thus the angle of the linear polarization in the last RF has to change by the same amount. Figure D.1 shows the predicted value of $p$ on July 21 with $\gamma=50^{\circ}$ and the corresponding value of $\beta_{\text {in }}-\beta_{\text {out }}$ calculated using the telescope model. The deviation between both does not exceed the level of numerical inaccuracies due to the calculation of the inverse of trigonometric functions in the derivation of the angles of incidence and rotation angles.

\section{Appendix E: Telescope matrix}

Figure E.1 displays the Mueller matrix of the telescope on two dates: March $21\left(\delta_{\odot} \sim 0^{\circ}\right.$, black $)$ and July $28\left(\delta_{\odot} \sim 20^{\circ}\right.$, grey $)$ for both visible $(630 \mathrm{~nm}$, solid) and infrared $(1.5 \mu \mathrm{m}$, dotted $)$ spectral ranges. In March the first coelostat mirror, C1, is usually set at $\gamma=50^{\circ}$ in the morning, and $-50^{\circ}$ in the afternoon, to reduce the pillar height of the second mirror to about $1 \mathrm{~m}$.

For the visible spectral range the values $(n, k)=(1.41,7.6)$, for infrared $(n, k)=(1.466,16.118)$ were used. The cross talk might exceed $20 \%$ in the early morning for the visible wavelength, while in the infrared it is usually smaller.

Observations with $\gamma=0^{\circ}$ are generally to be preferred due to the reduction of the cross talk, which is induced by the coelostat in this configuration. But, depending on the wind speed, it may be preferable to reduce the image motion by moving the first coelostat mirror to $\gamma \neq 0^{\circ}$, which reduces the pillar height of the second mirror. 
C. Beck et al.: Polarization model for the VTT, Online Material p 5
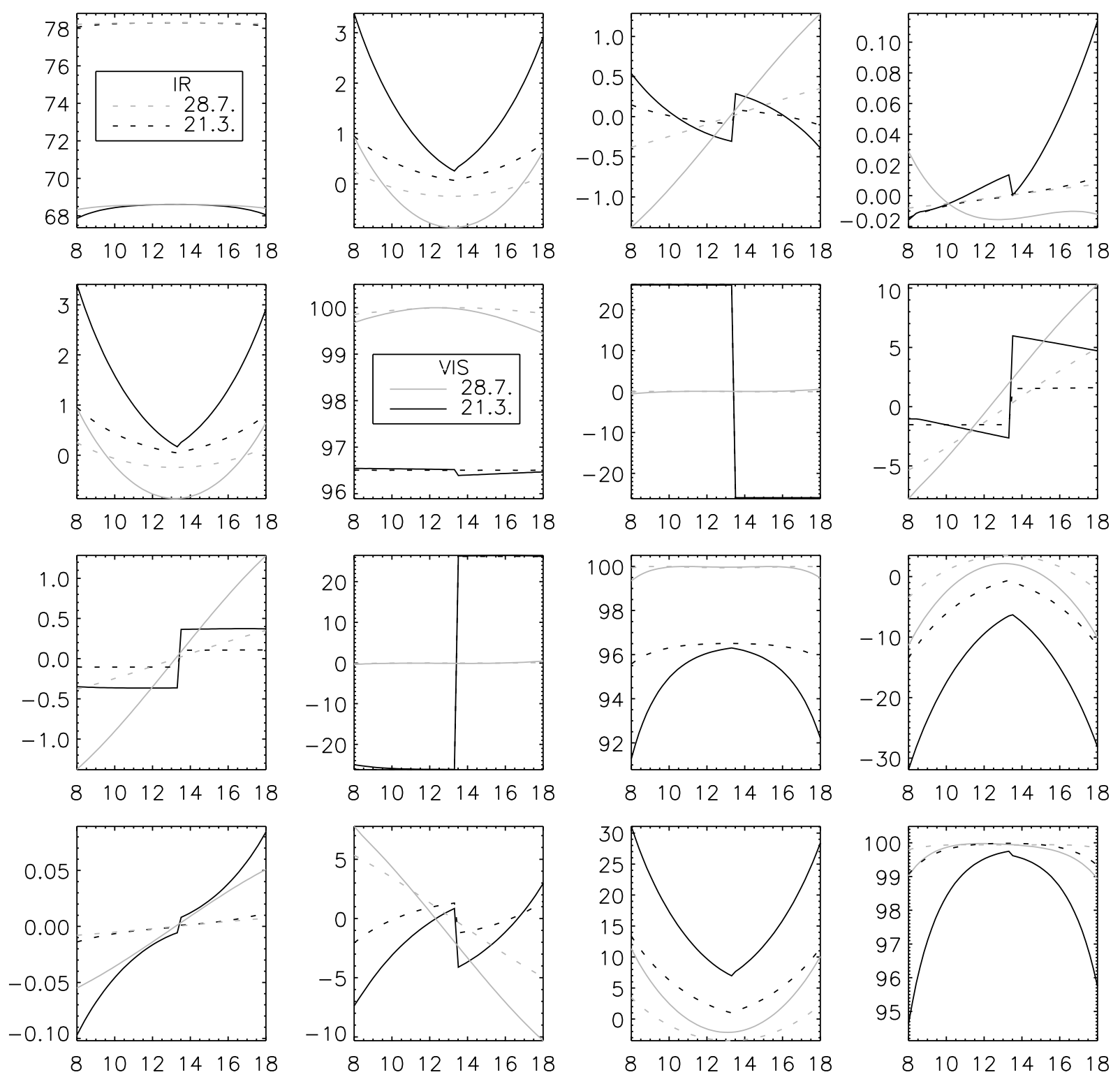

Fig. E.1. Daily variation of the 16 entries of the telescope Mueller matrix, $\mathcal{T}$, for two dates: March 21 (black, $\delta_{\odot} \sim 0^{\circ}, \gamma= \pm 50^{\circ}$ ) and July 28 (grey, $\delta_{\odot} \sim 20^{\circ}, \gamma=0^{\circ}$ ). Dotted: at $1.5 \mu \mathrm{m}$; solid: at $630 \mathrm{~nm}$. The ordinate gives Universal Time (UT) in hours. All cross talk entries are normalized with the total intensity transmission $\mathcal{T}(1,1)$ (upper left) and are given in $\%$. 